\title{
Memristive Cellular Automata for Modeling of Epileptic Brain Activity
}

\author{
Rafailia-Eleni Karamani*, Angelos Fyrigos*, Vasileios Ntinas*†, Ioannis Vourkas ${ }^{\ddagger}$, \\ Georgios Ch. Sirakoulis* and Antonio Rubio ${ }^{\dagger}$ \\ * Department of Electrical and Computer Engineering \\ Democritus University of Thrace, Xanthi, Greece \\ ${ }^{\dagger}$ Department of Electronic Engineering \\ Universitat Polytécnica de Catalunya, Barcelona, Spain \\ $\ddagger$ Department of Electronic Engineering \\ Universidad Técnica Federico Santa María, Valparaíso, Chile \\ Emails: rafakara@ee.duth.gr, ifyrigos@ee.duth.gr, vntinas@ee.duth.gr, ioannis.vourkas@usm.cl, \\ gsirak@ee.duth.gr, antonio.rubio@upc.edu
}

\begin{abstract}
Cellular Automata (CAs) is a nature-inspired and widespread computational model which is based on the collective and emergent parallel computing capability of units (cells) locally interconnected in an abstract brain-like structure. Each such unit, referred as $\mathrm{CA}$ cell, performs simplistic computations/processes. However, a network of such identical cells can exhibit nonlinear behavior and be used to model highly complex physical phenomena and processes and to solve problems that are highly complicated for conventional computers. Brain activity has always been considered one of the most complex physical processes and its modeling is of utter importance. This work combines the $\mathrm{CA}$ parallel computing capability with the nonlinear dynamics of the memristor, aiming to model brain activity during the epileptic seizures caused by the spreading of pathological dynamics from focal to healthy brain regions. A CAbased confrontation extended to include long-range interactions, combined with the recent notion of memristive electronics, is thus proposed as a modern and promising parallel approach to modeling of such complex physical phenomena. Simulation results show the efficiency of the proposed design and the appropriate reproduction of the spreading of an epileptic seizure.
\end{abstract}

Keywords-Cellular Automata, memristor, modeling, SPICE, epilepsy.

\section{INTRODUCTION}

Efficient modeling of biological systems and processes using conventional computing architectures is a priori impossible, which is something well known, since the latter function in a completely different manner than the hardware (HW) normally used for this task. For example, the most widespread computer architecture, namely the von Neumann architecture, keeps the processing unit separated from memory, while in nature processing and memory are considered unified notions. However, there are various unconventional computing approaches that comply with this unified functioning requirement. For instance, the Cellular Automata (CAs), a biologically-inspired parallel computing architecture proposed a long time ago also by von Neumann [1], is a model capable of capturing the characteristics of any high-order complex system by using a network of smaller identical systems, the CA cells. Owing to their spatial interconnections which permit parallel local interactions, and besides being simplistic, the CA cells demonstrate emerging dynamics required to model more complex systems. As a result, such collective behavior enables an effective description of a variety of physical phenomena and provides an alternative solution to rather difficult computational problems.

Speaking of biological systems, nowadays, the human brain is a case whose complete understanding and the further modeling and emulation of its functioning constitutes one of the utter importance major scientific challenges. As a more specific example, we refer to the epileptiform brain activity, an important field of study owing to the impact it has over several neuronal layers. It is characteristic that even an electroencephalograph (EEG) is not able to completely capture the brain activity during an epileptic seizure, but only a collective effect of just some neuronal clusters [2]. Several studies have applied different computational techniques aiming to model, simulate, and emulate the epileptiform brain activity, towards the prediction or even the entire prevention of epileptic seizures. For instance, in [2] the epileptiform brain activity in the neocortex was simulated by varying the ratio between excitatory and inhibitatory cell types, while also considering the synchronization effects produced by gap junctions. Moreover, Chiu et al. in [3] aimed to the prediction of the onsets of a epileptic seizure by training artificial neural networks (ANN). Likewise, Tetzlaff et al. in [4] used cellular neural networks (CNN) and utilized their high parallelism to effectively characterize the spatio-temporal properties of epileptic seizures, whereas in [5] a discrete time CNN implementation was presented, able to predict a potential incoming seizure. Further, the potentiality of highly scalable CA-based simulations of neural activity was discussed in [6], where an event-driven framework was used instead of computationally costly continuous simulation engines.

In this direction, this paper presents a modern memristive CA-based confrontation of the modeling of epileptic brain activity. Memristor (short for memory resistor) is a novel nanoelectronic device, highly promising for the design of innovative computing architectures. After the recent presentation of the first "modern" memristor by Hewlett-Packard (HP) Labs [7], which connected for the first time the 1971 Chua's theory [8] with practice, an ever increasing interest in memristive 


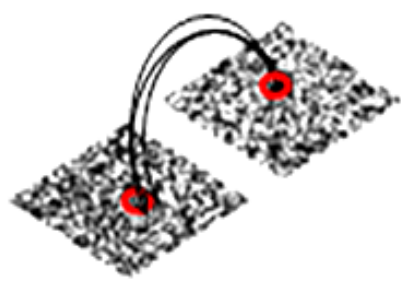

(a)

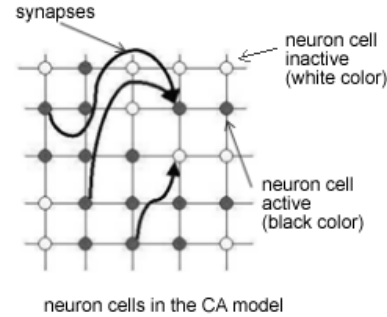

(b)

Fig. 1. (a) Two CA grids of neuron-cells with local and long-range connections, as presented in [16]. One grid corresponds to a healthy region and the other to the pathological region of the brain. (b) A magnified CA grid where the black (white) dots correspond to active (quiet) sites [16].

technology and its applications quickly emerged. More specifically, the memristor is a two-terminal analog device of variable resistance (also referred to as memristance), affected by the history of the voltage applied across its terminals. It is a novel circuit element which enriches the toolcase of circuit designers and researchers, as it can introduce memory capabilities and programmability in a vast amount of circuits and systems [9], as well as lead a whole new era of research on beyond von Neumann architectures and in-memory computing techniques [10], [11].

Itoh and Chua [12] introduced for the first time the use of memristors in CA configurations applied to several computational systems, at a more theoretical level. More practical implementations of memristive CA were later proposed for shortest-path computations, pseudorandom number generation and biomedical applications [13], [14], [15]. The proposed here novel memristive CA cell structure was envisaged from previous work in [14] and the presented simulation results proved its suitability for the simulation of complex grids of interconnected neurons, where the propagation of epileptiform brain activity between healthy and focal brain regions was correctly predicted.

\section{Modeling Epilepsy Propagation with CA}

Owing to its inherent nature-like functionality, Cellular Automata (CA) is a tool suitable for the depiction of complex biological processes and structures by electronic hardware (HW). Moreover, as previously shown in [16], the electric activity of healthy and pathological brain regions can be modeled using CA. In this case, a well-designed CA would map the interactions of brain neurons to a proper spatial network of statistically identical cells, each one of which can be described by a threshold-controlled nonlinear module. From the theoretical perspective of CA, this kind of system requires a CA cell state $\mathbf{C}(i, t)=\{0,1\}$, which shows the firing state of a neuron, and a state transition rule expressed as follows:

$$
\mathbf{C}(i, t+1)=\mathbf{R}\left(\mathbf{C}(i, t), \mathbf{C}\left(n_{1}^{i}, t\right), \ldots, \mathbf{C}\left(n_{k}^{i}, t\right), E x c\right)
$$

In (1), the $\mathbf{C}\left(n_{j}^{i}, t\right)$ represents the cell state of the $j^{\text {th }}$ neighbor of the cell $i$ at time $t$, whereas Exc corresponds to any excitation signal which originates from a brain region

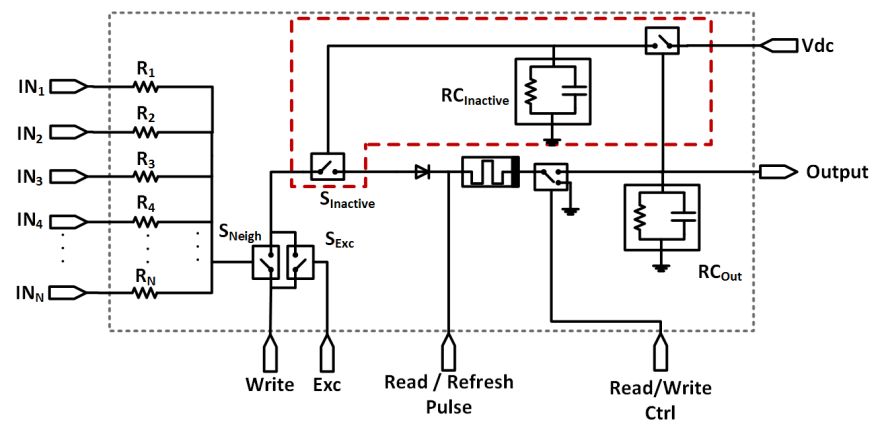

Fig. 2. Circuit-level representation of the proposed memristor-based CA cell.

outside the simulated system. The number of neighbors $(k)$ can differ between cells, according to the required connectivity for this kind of system which does not follow any of the wellknown CA neighborhoods (e.g. Moore's or von Neumann's). Particularly, in this work the simulated system consists of two $\mathrm{CA}$ grids, $\mathrm{M} \times \mathrm{N}$ each, where every cell may receive input signals from any other cell of the same grid, but only a small number of cells are connected with cells of the other grid. This topology is shown in Fig. 1 [16]. According to the rule $\mathbf{R}$, every cell can be activated (i.e. be in state $\mathbf{C}(i, t)=1$ ) in two ways: (i) when the aggregate input signal received from its neighbors exceeds a certain threshold, or (ii) if the Exc signal exceeds a certain threshold (thresholds for case (i) and (ii) may have different values). When activated, the cell produces a spike-like output signal, which turns high $\left(\operatorname{logic}^{\prime} 1^{\prime}\right)$ for a short excitation period and then remains low $\left(\operatorname{logic}{ }^{\prime} 0^{\prime}\right)$ during a refractory period (here refractory lasts longer than excitation). A more detailed circuit-level description of CA cell operation is given in the following Section.

Furthermore, following the original description in [16], it is important to consider that the maximum number of incoming synaptic connections to every cell during an epileptic seizure phase, is different in the two CA grids. For example, in a healthy phase, all cells in both grids can have at most $Z_{\text {healthy }}$ incoming synapses. However, during the epileptic seizure phase the incoming synaptic connections of the pathogenic grid's cells can be reduced to $Z_{\text {epilepsy }}$, where $Z_{\text {epilepsy }}<Z_{\text {healthy }}<M \times N$. Moreover, the external excitation signal Exc is produced randomly both for the healthy and the pathogenic grid during the healthy phase. However, the epileptic seizure causes instead a synchronized chaotic Exc signal to the pathogenic grid, which then exhibits a much higher brain activity.

\section{Memristive CA Cell Design}

The proposed here memristive CA cell was inspired by the previous theoretical background laid by Itoh and Chua in [12]. However, unlike [12] where the memristor dynamics vary according to the requirements of every target application, here the cell was developed at circuit-level having in mind its universality and resulting to the corresponding novel circuit design. Therefore, a single behavioral threshold-based and SPICE-compatible [17] model of a voltage-controlled bipolar memristor with linear ON and OFF states, was used, thus paving the way towards potential real system implementations using available memristor devices [18] in the near future. 


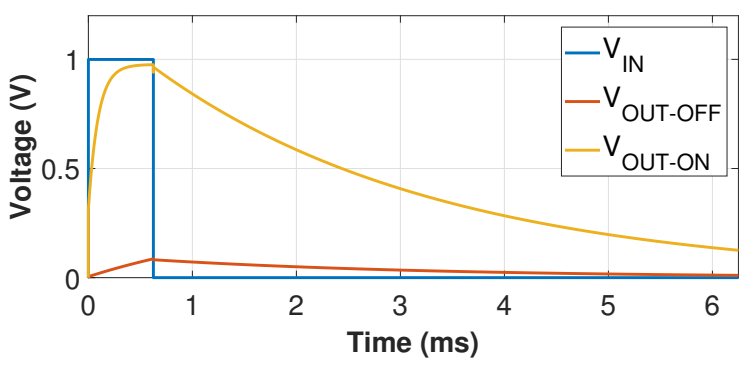

Fig. 3. Input read pulse and cell's output response for cell state $\mathbf{C}=0$, (red line) and $\mathbf{C}=1$ (yellow line).

The aforementioned device model [17] used in this work is a general behavioral model, is SPICE-compatible and, most importantly, easy-enough to configure in order to capture the basic characteristics of both filamentary and interfacial switching devices [19]. Hence it was found suitable for the purposes of our application. In specific, the device dynamics are described by the following set of equations:

$$
\begin{aligned}
& I_{M}(x, t)=G(x) \cdot V_{M}(t) \\
& M(x)=\frac{1}{G(x)}=f_{0} \cdot \frac{e^{2 x}}{x} \\
& x=x_{0} \cdot\left(1-\frac{m}{r}\right) \\
& \dot{r}= \begin{cases}a_{R S T} \cdot \frac{V_{M}+V_{R S T}}{c+\left|V_{M}+V_{R S T}\right|} & , V_{M}<V_{R S T} \\
\beta \cdot V_{M}, & , V_{R S T} \leq V_{M} \leq V_{S} \\
a_{S} \cdot \frac{V_{M}-V_{S}}{c+\left|V_{M}-V_{S}\right|} & , V_{M}>V_{S}\end{cases}
\end{aligned}
$$

A detailed description of the physical correspondence of the above equations and the role of each parameter can be found in [20]. The set of parameter values used in all simulations presented next, are: $f_{0}=310$ a.u., $L_{0}=5$ a.u., $m=82$ a.u., $\alpha_{S}=\alpha_{R S T}=-3 \times\left(10^{8}\right)$ a.u., $\beta=-10$ a.u., $c=0.1$ a.u., $\left|V_{S}\right|=\left|V_{R S T}\right|=1.5 \mathrm{~V}$, and $\left[R_{O N}, R_{O F F}\right]=$ $[1.8,211] k \Omega$.

Fig. 2 presents the memristive CA cell structure. Owing to the resistive switching property of memristor, one of the key characteristics of CA, namely the cell state, here it is mapped to memristance values. Consequently, a read-out mechanism is necessary to properly convert the current memristance to an output signal that can be communicated to the neighboring cells. The discrete time steps of CA evolution here comprise two phases, the Reading phase and the Writing phase. In the Reading phase, the Read/Write Ctrl switch connects the memristor to the output node and drives the reading pulse to it according to the voltage divider between the memristor and the $R C_{O U T}$ (see Fig. 2). The $R C_{O U T}$ is a resistorcapacitor pair which acts as a RC delay module, aiming to maintain the output voltage during the Writing phase. The values of $R C_{\text {out }}$ elements are properly selected in order to achieve easily distinguishable output voltage levels for the cells, corresponding to high resistance $\left(R_{O F F}\right)$ and low resistance $\left(R_{O N}\right)$ memristor states. Furthermore, the applied Reading pulse amplitude is selected lower than the memristor's voltage thresholds $\left(V_{\text {SorRST }}\right)$, so that it does not affect the current memristive state, but also high enough to generate an

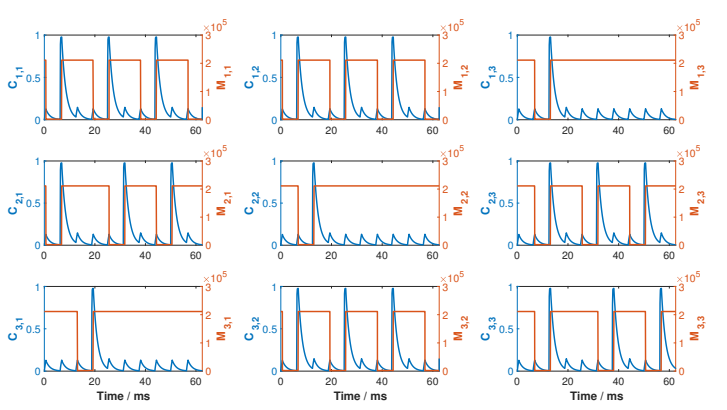

(a)

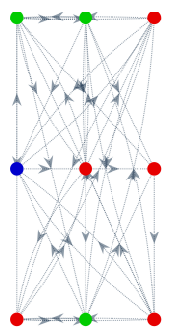

(b)

Fig. 4. (a) Output voltage (blue) and memristance (orange) evolution in time of every cell in a $3 \times 3$ grid such as the one shown in (b).

output response sufficient to affect the neighboring cells. Fig. 3 shows the input Reading pulse and the corresponding response of a cell when the state memristor is in $R_{O F F}(\mathbf{C}=0)$ and in $R_{O N}(\mathbf{C}=1)$, respectively.

After the Reading phase, the Read/Write Ctrl switch changes to the Writing phase configuration, in which one memristor's terminal is connected directly to the ground. In this phase, any input signal drops on the memristor's terminals, so its state is modified. The duration of the Writing phase is selected larger than the Reading phase, because of the required discharge time of $R C_{O U T}$ and the time it takes the memristor to switch its state. In the Writing phase, the CA state transition Rule is used and the input signal applied to the memristor modifies its memristance accordingly. In the proposed cell in Fig. 2, the state transition Rule was implemented using a passive analog adder based on Millman's theorem [21]. In fact, all the input signals originating from the neighboring cells are connected to a system of equal series resistances whose common node controls the $S_{\text {Neigh }}$ switch. So, when the common node voltage exceeds a threshold, then a positive voltage (Write) is applied to the top terminal of the memristor, while the bottom terminal is grounded. However, regardless of the state of the neighbors, a negative signal (Refresh) is also applied to the top terminal of the memristor in every Writing phase of a CA step (with exactly the same timing as the Write signal) which resets the device memristance to $R_{O F F}$. Nevertheless, if the $S_{\text {Neigh }}$ switch is closed, then Write overpowers Refresh and memristance is set instead to $R_{O N}$. Furthermore, an external signal Exc controls the $S_{E x c}$ switch, which is connected in parallel to $S_{N e i g h}$ and can active the cell regardless of the neighbors' state, depending on external stimuli, i.e. signals considered to be received from outside the simulated brain regions. Finally, a diode prevents the Read signal from being connected to the ground accidentally when Write signal is zero and switches $S_{\text {Neigh }}, S_{E x c}$ and $S_{\text {Inactive }}$ are closed.

So far, and as shown in Fig. 2, the state transition Rule depended only on the states of the neighboring cells. Nevertheless, in order to properly model healthy and epileptiform brain areas using the CA described in Section II, an additional feature was introduced to keep the cell inactive during a predefined time period after its activation, corresponding to the excitation and the refractory times of a real neuron. This consisted in a second delay module, namely $R C_{\text {Inactive }}$ (red dotted area in Fig. 2). After the activation of a cell, the 


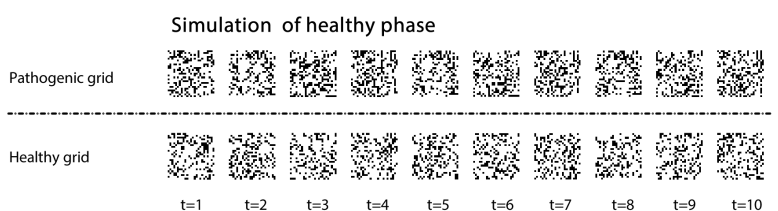

(a)

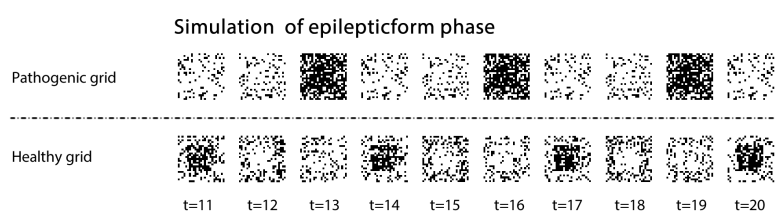

(b)

Fig. 5. Ten consecutive time-steps of pathogenic and healthy brain region evolution during (a) healthy and (b) epileptiform phases.

capacitor of this RC module is charged by the source $V_{D C}$ and opens the $S_{\text {Inactive }}$ switch so that Write cannot affect the memristor. The characteristics of this delay module were properly chosen to keep the cell inactive for two time-steps after one activation. Moreover, except for the signals $I N_{1,2, \ldots, N}$ and Output, which are used for the cell interconnection, the rest of the cell's external signals shown in Fig. 2 are universal signals common for every cell of the grid. Particularly, Write, $V_{D C}$ and Read/WriteCtrl are generated by three different sources which are connected to every cell of the grid, likewise it happens for Refresh and Read. Regarding Exc, this can be universal for all cells or not. The circuit-level development of the proposed CA cell as well as the performance of the entire CA model were studied using SPICE simulations, whose results are presented in the following Section.

\section{Memristive CA Modeling of Healthy AND EPILEPTIFORM BRAIN PHASES}

First of all, regarding CA cell interconnections, a full random intra-grid and inter-grid connection pattern was applied, to comply with the unpredictable synaptic connectivity of biological neurons. The mean value of the inputs received by every cell (i.e. the outputs of the connected neighbors) controls the Write switch, whose threshold value is proportional to the number of inputs. An example of a $3 \times 3 \mathrm{CA}$ grid is shown in Fig. 4 for readability reasons. Every subplot of Fig. 4a shows the output voltage and memristance evolution in time of the corresponding cell in the topology shown in Fig. 4b. Every cell has 4-5 neighbors and if at least 3 of them are active, then it gets activated. In this example, the green cells are permanently excited via Exc signal, the blue is excited only in the very first time-step (initialized with $\mathbf{C}=^{\prime} 1^{\prime}$ ), whereas the state of the rest depends only on their neighbors. In Fig. 4a, the effect of $R C_{\text {Inactive }}$ is notable as all cells after being activated they remain inactive for two time-steps. The selected time constants of the delay modules were set as $t_{O u t}=2.7 \mathrm{~ms}$ and $t_{\text {Inactive }}=9 \mathrm{~ms}$.

During the epileptiform phase, the cells in the pathogenic grid lose some of their synaptic connections in a random manner. At circuit level, this is achieved via a switch that is connected to the inputs of the cell. Additionally, a chaotic signal is connected to the Exc input of every cell in the

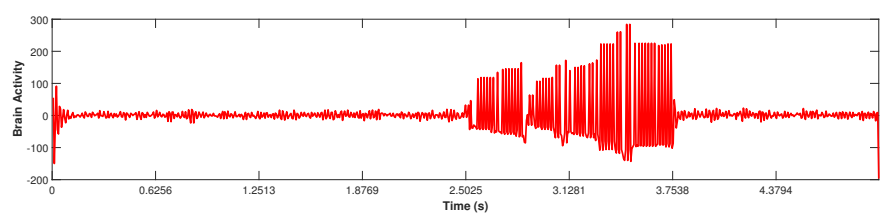

(a)

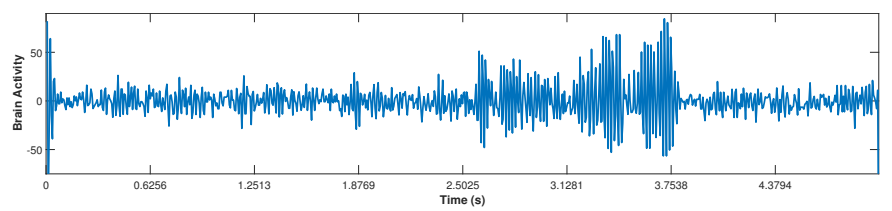

(b)

Fig. 6. Aggregate brain activity evolution in time for the (a) pathogenic and (b) the healthy brain region.

entire pathogenic region which increases the local activity. On the contrary, the Exc input in connected to a randomized amplitude noise source during the healthy phase. Two $25 \times 25$ memristive CA grids were designed and simulated in SPICE complying with the aforementioned requirements. A random integer between 2 and 7 intra-grid synaptic connections was used for all cell in both grids, whereas six inter-grid synaptic connections were added only in the central $12 \times 12$ area of each grid. In this CA configuration, the $S_{N e i g h}$ and $S_{E x c}$ thresholds were chosen as 0.5 of the maximum value, meaning that activation occurs either when half of the neighbors are active or if Exc exceeds $0.5 \mathrm{~V}$ (as the external random or chaotic voltages are normalized in the range $[0,1])$. Fig. 5 shows the cell states of both grids for 10 consecutive time-steps of each phase (healthy and epileptiform). For readability reasons, the state of every cell is registered at the end of the read pulse and the output voltage is mapped to either black (active) or white (inactive) pixels. Most importantly, through longer lasting simulations, the epileptic seizure propagation from the pathogenic to the healthy region was correctly captured. For its study, the sum of the active cells of every grid was computed and used as a metric of the amount of local brain activity. Additionally, a high-pass filter was applied to the brain activity evolution in time to remove the mean value (DC offset) and present fluctuations in a clearer manner. In Fig. 6 it can be observed that, during the epileptiform phase, the increased brain activity of the pathogenic region is highly affecting the healthy region, where epilepsy-like phenomena are emerging.

\section{CONClusions}

This paper presented a novel memristive Cellular $\mathrm{Au}-$ tomata circuit-level approach for the modeling of healthy and pathogenic brain regions during epileptic seizures. The proposed design emulates epilepsy-related phenomena in the brain, exploiting the parallelism of CA and memristor's dynamics to model a highly scalable network of neurons. Further study on the timing capabilities of the system is under investigation, including optimization of power consumption.

\section{ACKNOWLEDGMENT}

This work was supported in part by the Chilean government under the research grants CONICYT FONDECYT Postdoctorado No. 3160042/2016. 


\section{REFERENCES}

[1] J. von Neumann, Theory of Self-reproducing Automata. University of Illinois Press, 1966.

[2] W. van Drongelen, H. C. Lee, M. Hereld, D. Jones, M. Cohoon, F. Elsen, M. E. Papka, and R. L. Stevens, "Simulation of neocortical epileptiform activity using parallel computing," Neurocomputing, vol. 58, pp. 1203 1209, 2004.

[3] A. W. Chiu, S. Daniel, H. Khosravani, P. L. Carlen, and B. L. Bardakjian, "Prediction of seizure onset in an in-vitro hippocampal slice model of epilepsy using gaussian-based and wavelet-based artificial neural networks," Annals of biomedical engineering, vol. 33, no. 6 , pp. 798-810, 2005.

[4] R. Tetzlaff, R. Kunz, C. Ames, and D. Wolf, "Analysis of brain electrical activity in epilepsy with cellular neural networks (cnn)," signal, vol. 400, no. 200, p. 0, 1999.

[5] F. Gollas, C. Niederhofer, and R. Tetzlaff, "Prediction of brain electrical activity in epilepsy using a higher-dimensional prediction algorithm for discrete time cellular neural networks (dtcnn)," in Circuits and Systems, 2004. ISCAS'04. Proceedings of the 2004 International Symposium on, vol. 5. IEEE, 2004, pp. V-V.

[6] E. T. Claverol, A. D. Brown, and J. E. Chad, "A large-scale simulation of the piriform cortex by a cell automaton-based network model," IEEE transactions on biomedical engineering, vol. 49, no. 9, pp. 921-935, 2002.

[7] D. B. Strukov, G. S. Snider, D. R. Stewart, and R. S. Williams, "The missing memristor found," Nature, vol. 453, no. 7191, pp. 80-83, 2008.

[8] L. Chua, "Memristor-the missing circuit element," IEEE Transactions on circuit theory, vol. 18, no. 5, pp. 507-519, 1971.

[9] M. D. Ventra and Y. V. Pershin, "Just add memory," Sci. American, vol. 312, pp. 56-61, Feb. 2015.

[10] J. J. Yang, D. B. Strukov, and D. R. Stewart, "Memristive devices for computing," Nat. Nano, vol. 8, pp. 13-24, 2013.

[11] I. Vourkas and G. Sirakoulis, Memristor-Based Nanoelectronic Computing Circuits and Architectures: Foreword by Leon Chua. Springer, 2015, vol. 19.

[12] M. Itoh and L. O. Chua, "Memristor cellular automata and memristor discrete-time cellular neural networks," Int. J. Bifurcation Chaos, vol. 19, no. 11, pp. 3605-3656, 2009.

[13] D. Stathis, I. Vourkas, and G. C. Sirakoulis, "Shortest path computing using memristor-based circuits and cellular automata," Springer LNCS, vol. 8751, pp. 398-407, 2014.

[14] R. E. Karamani, V. Ntinas, I. Vourkas, and G. C. Sirakoulis, "1-D Memristor-based Cellular Automata for Pseudo-Random Number Generation," in International Symposium on Power and Timing Modeling, Optimization, and Simulation (PATMOS), 2017.

[15] J. Secco, M. Farina, D. Demarchi, F. Corinto, and M. Gilli, "Memristor cellular automata for image pattern recognition and clinical applications," in IEEE International Symposium on Circuits and Systems (ISCAS), 2016, pp. 1378-1381.

[16] V. Tsoutsouras, G. C. Sirakoulis, G. P. Pavlos, and A. C. Iliopoulos, "Simulation of healthy and epileptiform brain activity using cellular automata," International Journal of Bifurcation and Chaos, vol. 22, no. 09, p. 1250229, 2012.

[17] I. Vourkas, A. Batsos, and G. C. Sirakoulis, "Spice modeling of nonlinear memristive behavior," Int. J. of Circuit Theory and Applications, vol. 43 , no. 5 , pp. 553-565, 2015.

[18] Neuromemristive artificial intelligence. [Online]. Available: https://knowm.org

[19] G. Sassine, S. La Barbera, N. Najjari, M. Minvielle, C. Dubourdieu, and F. Alibart, "Interfacial versus filamentary resistive switching in $\mathrm{TiO} 2$ and HfO2 devices," Journal of Vacuum Science \& Technology B, vol. 34, no. 1, p. 012202, 2016.

[20] I. Vourkas and G. C. Sirakoulis, "A novel design and modeling paradigm for memristor-based crossbar circuits," IEEE Transactions on Nanotechnology, vol. 11, no. 6, pp. 1151-1159, 2012.

[21] J. Millman, "A useful network theorem," Proceedings of the IRE, vol. 28, no. 9, pp. 413-417, 1940. 